\title{
Abkürzungsverzeichnis der Museen, Sammlungen und Institutionen
}

Forschst. Magd. Forschungsstelle Magdeburg des Zentralinstituts für Alte Geschichte und Archäologie der Akademie der Wissenschaften der DDR, 1086 Berlin, Leipziger Straße 3-4

Inst. Berlin Zentralinstitut für Alte Geschichte und Archäologie, Bereich Ur- und Frühgeschichte der Akademie der Wissenschaften der DDR, 1086 Berlin, Leipziger Straße 3-4

Inst. Greifswald Sektion Geschichtswissenschaft der Ernst-Moritz-Arndt-Universität, Bereich Ur- und Frühgeschichte, 2200 Greifswald, Domstraße 11

Inst. Jena Vorgeschichtliches Museum der Friedrich-Schiller-Universität Jena, Sektion Geschichte, Bereich Ur- und Frühgeschichte, 6900 Jena, Ernst-Thälmann-Ring 24 a

Mus. Altenburg Schloßmuseum, 7400 Altenburg, Schloß 2

Mus. Bautzen Stadtmuseum Bautzen, Museum für Kunst- und Kulturgeschichte, 8600 Bautzen, Platz der Roten Armee 1 a

Mus. Berlin (West)

Mus. Dresden

Museum für Vor- und Frühgeschichte, Berlin 19, Schloß Charlottenburg, Langhansbau Landesmuseum für Vorgeschichte, Forschungsstelle für die Bezirke Dresden, Karl-MarxStadt und Leipzig, 8060 Dresden, Karl-Marx-Platz - Japanisches Palais

Mus. Erfurt

Mus. Frankfurt

Mus. Halle Angermuseum, 5020 Erfurt, Anger 18 Bezirksheimatmuseum für den Bezirk Frankfurt (Oder), 1200 Frankfurt (Oder), Ph.E.-Bach-Straße 11

Mus. Leipzig

Mus. Potsdam Landesmuseum für Vorgeschichte, Forschungsstelle für die Bezirke Halle und Magdeburg, 4020 Halle (Saale), Richard-Wagner-Straße 9/10

Naturwissenschaftliches Museum, 7010 Leipzig, Lortzingstraße 3

Museum für Ur- und Frühgeschichte Potsdam, Forschungsstelle für die Bezirke Potsdam, Frankfurt und Cottbus, 1502 Potsdam-Babelsberg, Schlo $B$ Babelsberg

Mus. Rostock Museum der Stadt Rostock, Bezirksmuseum für den Westteil des Bezirks Rostock, 2500 Rostock, August-Bebel-Straße 1

Mus. Schwerin Museum für Ur- und Frühgeschichte Schwerin, Forschungsstelle für die Bezirke Rostock, Schwerin und Neubrandenburg, 2750 Schwerin, Schloß

Mus. Stralsund Kulturhistorisches Museum Stralsund, Bezirksmuseum für den Ostteil des Bezirks Rostock, 2300 Stralsund, Mönchstraße 25-27

Mus. Waren Müritz-Museum Waren, Bezirksmuseum des Bezirkes Neubrandenburg, 2060 Waren (Müritz), Friedensstraße 5

Mus. Weimar Museum für Ur- und Frühgeschichte Thüringens, Forschungsstelle für die Bezirke Erfurt, Gera und Suhl, 5300 Weimar, Humboldtstraße 11

Staatl. Mus. Berlin Staatliche Museen zu Berlin, Museum für Ur- und Frühgeschichte, 1020 Berlin, Bodestraße 1-3

Staatl. Mus. Schwerin Staatliches Museum Schwerin, 2750 Schwerin, Am Alten Garten 
